\title{
Refraction of Surface Gravity Waves by Shear Waves
}

\author{
Stephen M. Henderson and R. T. GuZa \\ Scripps Institution of Oceanography, La Jolla, California \\ STEVE Elgar \\ Woods Hole Oceanographic Institution, Woods Hole, Massachusetts \\ T. H. C. Herbers \\ Department of Oceanography, Naval Postgraduate School, Monterey, California
}

(Manuscript received 8 November 2004, in final form 15 August 2005)

\begin{abstract}
Previous field observations indicate that the directional spread of swell-frequency (nominally $0.1 \mathrm{~Hz}$ ) surface gravity waves increases during shoreward propagation across the surf zone. This directional broadening contrasts with the narrowing observed seaward of the surf zone and predicted by Snell's law for bathymetric refraction. Field-observed broadening was predicted by a new model for refraction of swell by lower-frequency (nominally $0.01 \mathrm{~Hz}$ ) current and elevation fluctuations. The observations and the model suggest that refraction by the cross-shore currents of energetic shear waves contributed substantially to the observed broadening.
\end{abstract}

\section{Introduction}

Surface gravity waves approaching a straight coastline refract over the sloping seabed. According to Snell's law, both the mean wave angle (mean departure of wave propagation directions from shore normal) and the directional spread (range of wave angles) decrease with decreasing depth. Although the predicted decrease in mean angle is observed, the observed directional spread sometimes increases during shoreward propagation across natural surf zones (Herbers et al. 1999). Directional spreading determines the length of wave crests (Longuet-Higgins 1957), and it affects the nonlinear forcing of infragravity $(0.001-0.050 \mathrm{~Hz})$ waves (Herbers et al. 1995; Henderson and Bowen 2003).

Nonlinearity causes deviations from Snell's law, but nonlinear triad interactions, as simulated by a spectral Boussinesq model (Herbers et al. 2003), do not explain the observed spreading. Numerical simulations (Öz-

Corresponding author address: Stephen M. Henderson, Scripps Institution of Oceanography, La Jolla, CA 92093-0209.

E-mail: shenders@coast.ucsd.edu
kan-Haller and Li 2003) predict that swell is refracted by low-frequency shear instabilities of the alongshore current, called shear waves (Bowen and Holman 1989; Oltman-Shay et al. 1989). The resulting slow changes in wave angle contribute to the directional spread calculated from long time series. We show that broadening observed on a natural beach is roughly consistent with refraction by the fluctuating currents of shear waves. The field site, instrumentation, and observations are described in section 2. Consistent with previous observations (Herbers et al. 1999), the directional spread decreased as waves shoaled outside the surf zone, but often increased with decreasing depth inside the surf zone. A model relating directional broadening to refraction by low-frequency motions is derived (section 3). Given idealized swell and low-frequency currents, this model simplifies to a form analogous to Taylor's (1921) model of particle motion in turbulent flow (section 4). The observed broadening was roughly consistent with predictions of both the full and simplified models (section 5).

\section{Observations}

Wave directional properties were estimated using data collected on an ocean beach near Duck, North 
Carolina, during the 1997 SandyDuck experiment (Feddersen et al. 2000; Elgar et al. 2001; Herbers et al. 2003). Water pressures and horizontal velocities were measured (at $2 \mathrm{~Hz}$ ) for 4 months at 18 locations between the shore and 4-m depth (Fig. 1).

A mean wave angle at cyclic frequency $f$ is

$$
\theta(f)=\frac{1}{2} \tan ^{-1}\left[\frac{2 C_{u, v}(f)}{C_{u, u}(f)-C_{v, v}(f)}\right],
$$

where $u$ and $v$ are cross- and alongshore velocities, and $C_{\alpha, \beta}(f)$ is the cospectrum between $\alpha$ and $\beta$. The orientation $\theta(f)$ is the dominant principal axis of the $(u, v)$ cospectral matrix. A measure of the directional spread at $f$ is

$$
\sigma(f)=\left[\frac{C_{v^{\prime}, v^{\prime}}(f)}{C_{u^{\prime}, u^{\prime}}(f)+C_{v^{\prime}, v^{\prime}}(f)}\right]^{1 / 2},
$$

where $u^{\prime}=u \cos [\theta(f)]+v \sin [\theta(f)]$ is the velocity component in the mean direction $\theta(f)$, and $v^{\prime}$ is the velocity component perpendicular to $u^{\prime}$ (Longuet-Higgins 1957).

An energy-weighted mean angle of swell waves is (Herbers et al. 1999)

$$
\bar{\theta}=\frac{1}{2} \tan ^{-1}\left(\frac{2\langle\hat{u} \hat{v}\rangle}{\left|\left\langle\hat{u}^{2}\right\rangle-\left\langle\hat{v}^{2}\right\rangle\right|}\right),
$$

where the angle brackets denote a 3-h average, and $\hat{u}$ and $\hat{v}$ are cross-shore and alongshore velocities bandpassed between 0.05 and $0.15 \mathrm{~Hz}$. An energy-weighted directional spread for swell is

$$
\bar{\sigma}=\left(\frac{\left\langle\hat{v}^{\prime 2}\right\rangle}{\left\langle\hat{u}^{\prime 2}\right\rangle+\left\langle\hat{v}^{\prime 2}\right\rangle}\right)^{1 / 2},
$$

where $\hat{u}^{\prime}$ is the component of band-passed velocity in the direction $\bar{\theta}$, and $\hat{v}^{\prime}$ is the component of band-passed velocity perpendicular to $\hat{u}^{\prime}$.

Measured wave directional properties were similar to those observed on the same beach 3 years earlier (Herbers et al. 1999). Mean wave angles at the offshore, deepest instruments $\left(\bar{\theta}_{d}\right)$ usually were more oblique than at the onshore, shallowest instruments $\left(\bar{\theta}_{s}\right)$ (Fig. $2 c)$. Snell's law predicts that waves refract to conserve their alongshore wavelength $c /(f \sin \theta)$, where $c$ is the phase speed. For small-angle shallow-water waves, $\sin (\theta) \approx \theta$ and $c=(g h)^{1 / 2}$ (where $h$ is water depth), so Snell's law predicts that $\theta$ is proportional to $h^{1 / 2}$. The shoreward change in wave angle $\Delta \bar{\theta}=\bar{\theta}_{s}-\bar{\theta}_{d}$ was consistent (root-mean-square error $2.3^{\circ}$; see Fig. $2 \mathrm{~d}$ ) with the Snell's-law prediction:

$$
\Delta \bar{\theta}=\left[\left(\frac{h_{s}}{h_{d}}\right)^{1 / 2}-1\right] \bar{\theta}_{d}
$$
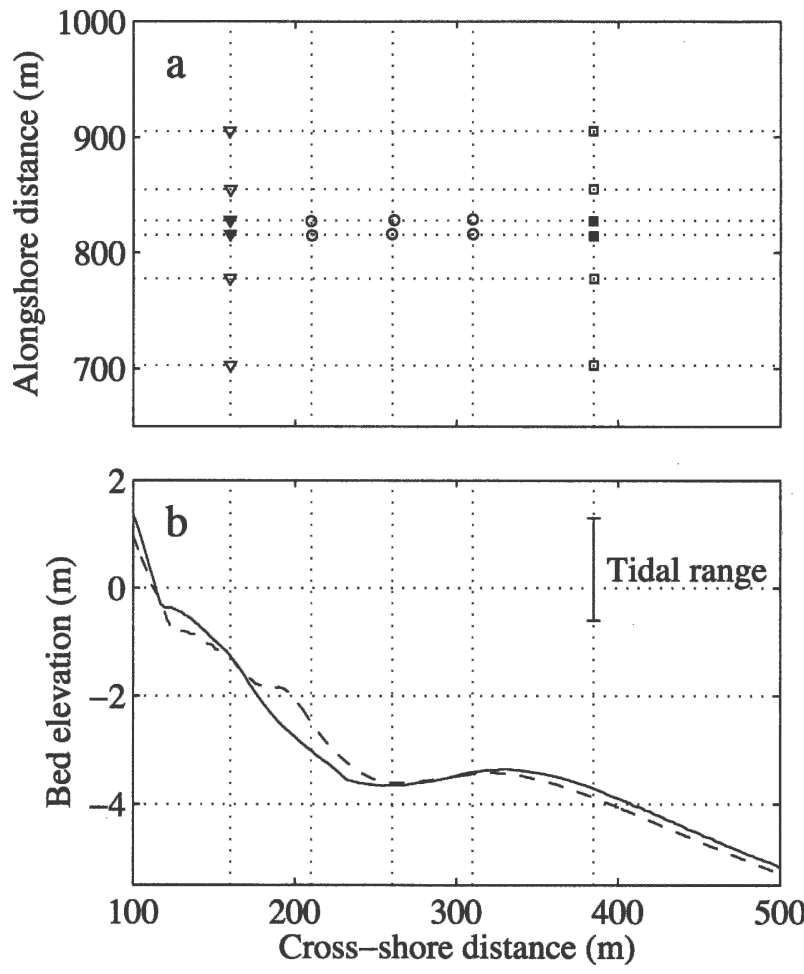

Fig. 1. (a) Plan view of instrument locations. Each symbol (defined in the text) represents a collocated pressure and velocity sensor. The Field Research Facility coordinate system is used. (b) Seabed elevations vs cross-shore distance on 13 August (solid curve) and 23 October (dashed curve). The average of four beach profiles measured between alongshore distances 695 and $915 \mathrm{~m}$ is shown.

where $h_{s}$ and $h_{d}$ are mean water depths at the shallow and deep locations, respectively. Snell's law also predicts directional narrowing:

$$
\Delta \bar{\sigma}=\left[\left(\frac{h_{s}}{h_{d}}\right)^{1 / 2}-1\right] \bar{\sigma}_{d}
$$

where $\Delta \bar{\sigma}=\bar{\sigma}_{s}-\bar{\sigma}_{d}$ is the change in spread between the deep and shallow locations. Sometimes narrowing was observed ( $\bar{\sigma}_{s}<\bar{\sigma}_{d}$, Fig. 2e), and both the observed and predicted changes in spread $\Delta \bar{\sigma}$ were negative (Fig. 2f). However, broadening $(\Delta \bar{\sigma}>0)$ also was observed (cases with $\Delta \bar{\sigma}>1^{\circ}$ are indicated with filled symbols in Fig. 2). For example, the observed $\Delta \bar{\sigma}$ exceeded $1^{\circ}$ during 3-5 September and 4-8 November, when the wave height in 8-m depth exceeded $2 \mathrm{~m}$ (Fig. 2a). These large waves broke far offshore, so dissipation was maximum [the ratio $q_{s} / q_{d}$ between linear theory swell energy fluxes (Herbers et al. 2000) at the shallow and deep instruments was much less than one; see Fig. 2b]. The energetic swell also generated strong low-frequency $(0.001-0.05 \mathrm{~Hz})$ motions. In high-energy conditions the kinetic energy of these motions $\left(h\left\langle u^{2}+v^{2}\right\rangle / 2\right)$ exceeded 

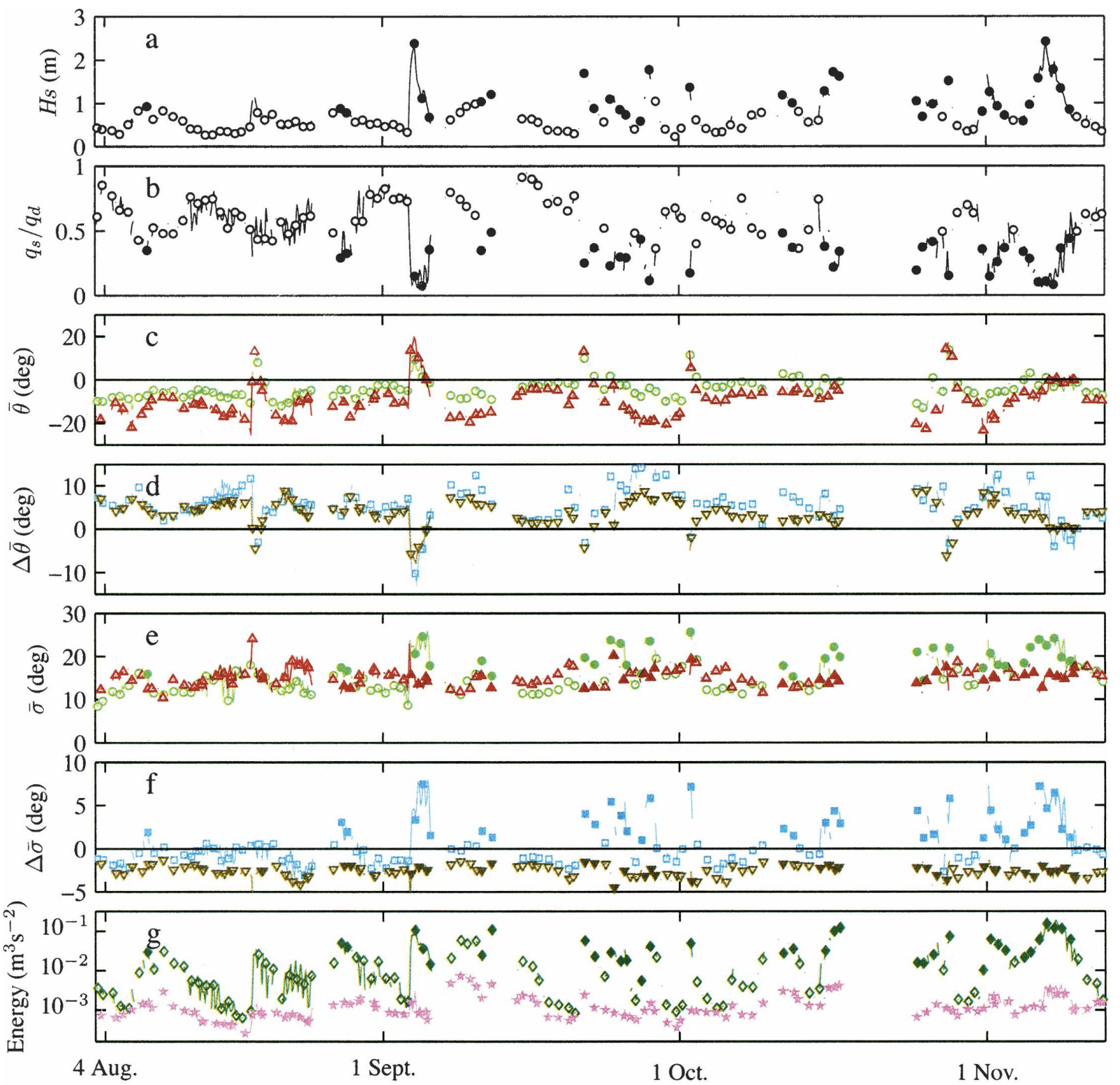

Time

FIG. 2. Time series of observed (a) significant wave height $H_{s}$ in 8-m water depth, (b) ratio $q_{s} / q_{d}$ of sea swell energy fluxes at the shallowest (most onshore) and deepest (most offshore) instruments (respective locations marked by filled triangles and filled squares in Fig. 1a), (c) mean wave angle [Eq. (3)] at the shallow $\left(\bar{\theta}_{s}\right.$, light green circles) and deep $\left(\bar{\theta}_{d}\right.$, dark red triangles) instruments, (d) change in mean angle $\Delta \bar{\theta}$ between deep and shallow instruments \{light blue squares, observed; dark brown triangles, Snell's law [Eq. (5)]\}, (e) directional spread [Eq. (4)] at the shallow $\left(\bar{\sigma}_{s}\right.$, light green circles) and deep ( $\bar{\sigma}_{d}$, dark red triangles) instruments, (f) change in spread $\Delta \bar{\sigma}$ between deep and shallow instruments \{light blue squares, observed; dark brown triangles, Snell's law [Eq. (6)]\} and (g) kinetic (dark green diamonds) and potential (light purple stars) energy of low-frequency $(0.001-0.05 \mathrm{~Hz})$ motions at the shallowest instruments. In all panels, symbols are plotted every $18 \mathrm{~h}$, and thin lines connect data points not shown with symbols. In (a), (b), (e), (f), and (g), filled symbols indicate occasions when $\Delta \bar{\sigma}>1^{\circ}$ (i.e., occasions when anomalous broadening was strong).

the potential energy $\left(g\left\langle\eta^{2}\right\rangle / 2\right.$, where $\eta$ is sea surface displacement) by one to two orders of magnitude (Fig. $2 \mathrm{~g}$, note the $\log$ scale), indicating that these motions were dominated by shear waves rather than gravity waves (Lippmann et al. 1999).
Swell mean angles, absolute spreads, and broadening, calculated by applying a direct estimation approach (Elgar et al. 1994) to arrays of pressure sensors at 1- and 4-m depths (squares and triangles in Fig. 1a), were consistent with the current-meter-based estimates pre- 
sented above, suggesting that current meter noise was not responsible for the estimated broadening [over the entire experiment, the rms error between pressure- and current-meter-based estimates of $\bar{\sigma}_{d}\left(\bar{\sigma}_{s}\right)$ was $1.2^{\circ}$ $\left.\left(2.7^{\circ}\right)\right]$

\section{Refraction by low-frequency motions}

Variations in the wave propagation direction $\theta$ are given by (Dean and Dalrymple 1991, section 4.8.2):

$$
\frac{\partial \theta}{\partial s}=-\frac{1}{c} \frac{\partial c}{\partial n},
$$

where $n$ and $s$ are along-crest and crest-normal coordinates, and the phase speed $c=\omega / k$ (where $\omega$ is the swell radian frequency and $k$ is the wavenumber) has been assumed to vary only over times much longer than a wave period.

The dispersion relation for linear waves is

$$
\left(\omega-\mathbf{k} \cdot \mathbf{u}_{L}\right)^{2}=g k \tanh \left[k\left(h+\eta_{L}\right)\right],
$$

where $\mathbf{k}$ is the wave vector (so $|\mathbf{k}|=k$ ), $g$ is gravitational acceleration, $h$ is mean depth, and $\mathbf{u}_{L}$ and $\eta_{L}$ are lowfrequency velocity and sea level fluctuations. In the small angle $[\theta=O(\varepsilon) \ll 1]$, Boussinesq $[k h=O(\varepsilon)]$ approximation with weak low-frequency motions $\left[|\mathbf{u}|_{L} /\right.$ $g h^{1 / 2}$ and $\eta_{L} / h$ both $\left.O\left(\varepsilon^{2}\right)\right]$, the phase speed

$$
c=c_{0}\left[1+\frac{u_{L}}{c_{0}}+\frac{\eta_{L}}{2 h}-\frac{(k h)^{2}}{6}+O\left(\varepsilon^{3}\right)\right],
$$

where $u_{L}$ is the cross-shore component of $\mathbf{u}_{L}$, and $c_{0}=$ $(g h)^{1 / 2}$ is the shallow-water phase speed (see the appendix). Low-frequency alongshore currents do not enter at this order because wave angles are small, so $\mathbf{k}$ is almost shore normal, and the Doppler shift $\mathbf{k} \cdot \mathbf{u}_{L}$ [Eq. (8)] is dominated by the cross-shore current.

Substituting Eq. (9) into Eq. (7), transforming into cross- $(x)$ and alongshore $(y)$ coordinates, and assuming a gently sloping, alongshore-uniform seabed yields (see the appendix)

$$
\begin{aligned}
\frac{\partial \theta}{\partial x}= & \frac{\theta}{2 h} \frac{\partial h}{\partial x}-\frac{\partial u_{L} / \partial y}{(g h)^{1 / 2}}-\frac{\partial \eta_{L} / \partial y}{2 h}-\frac{\partial \theta^{2} / \partial y}{2} \\
& +O\left(\varepsilon^{3}, \varepsilon^{2} \partial h / \partial x\right) .
\end{aligned}
$$

Taking the expected value $E$ of Eq. (10), and assuming statistical alongshore homogeneity $(\partial E[] / \partial y=0)$, yields an equation for the cross-shore evolution of the mean angle

$$
\frac{\partial E[\theta]}{\partial x}=\frac{E[\theta]}{2 h} \frac{\partial h}{\partial x} .
$$

The mean angle obeys Snell's law [Eq. (5)] because refraction by the fluctuating currents averages to zero. Multiplying both sides of Eq. (10) by

$$
\theta^{\prime}=\theta-E[\theta]
$$

and taking the expected value yields an equation for the cross-shore evolution of the angle variance:

$$
\frac{\partial E\left[\theta^{\prime 2}\right]}{\partial x}=\frac{E\left[\theta^{\prime 2}\right]}{h} \frac{\partial h}{\partial x}-\frac{2 E\left[\theta^{\prime} \partial u_{L} / \partial y\right]}{(g h)^{1 / 2}}-\frac{E\left[\theta^{\prime} \partial \eta_{L} / \partial y\right]}{h},
$$

where the fourth term on the right of Eq. (10) does not contribute because $E\left[\theta \partial \theta^{2} / \partial y\right]=2 E\left[\theta^{2} \partial \theta / \partial y\right]=(2 /$ 3) $\partial E\left[\theta^{3}\right] / \partial y=0$, by alongshore homogeneity. The wave angle variance, while still affected by bathymetric refraction [first term on the right of Eq. (13)], is modified by correlations between wave angles and low-frequency motions.

\section{Spreading by statistically homogeneous currents}

Next, the stochastic refraction model in Eq. (13) is illustrated using the idealized case of an initially plane wave $(\theta=0$ at $x=0)$ propagating in water of constant depth and sea level elevation $\left(\partial h / \partial x=0, \eta_{L}=0\right)$ with statistically homogeneous currents. The following resembles Taylor's (1921) analysis of particle motion in a turbulent flow, with wave angle here taking the place of particle displacement. From Eq. (11) the mean angle $E[\theta]=0$ for all $x$. For small $x$, fluctuations $\theta^{\prime}$ about $E[\theta]$ $=0$ are small, and Eq. (10) reduces to

$$
\frac{\partial \theta^{\prime}}{\partial x}=-\frac{\partial u_{L} / \partial y}{(g h)^{1 / 2}} .
$$

In a reference frame moving with the wave, $(g h)^{1 / 2} \partial / \partial x$ becomes $d / d t$, so shear rotates the angle of a given wave at the rate $d \theta^{\prime} / d t=-\partial u_{L} / \partial y$. Multiplying both sides of Eq. (14) by $2 \theta^{\prime}$ and averaging gives

$$
\frac{\partial E\left[\theta^{\prime 2}\right]}{\partial x}=-\frac{2 E\left[\theta^{\prime} \partial u_{L} / \partial y\right]}{(g h)^{1 / 2}},
$$

which also can be derived as a special case of Eq. (13). Initially (at $x=0$ ), waves are unidirectional and the covariance between wave angle $\theta^{\prime}$ and shear $\partial u_{L} / \partial y$ on the right of Eq. (15) vanishes. However, as waves are refracted by the shear, a nonzero covariance between wave angle and shear develops. From Eq. (14)

$$
\theta^{\prime}(x, t)=-\frac{1}{(g h)^{1 / 2}} \int_{0}^{x} \frac{\partial u_{L}\left[x^{\prime}, t-(g h)^{-1 / 2} x^{\prime}\right]}{\partial y} d x^{\prime},
$$


SO

$$
-\frac{2 E\left[\theta^{\prime} \partial u_{L} / \partial y\right]}{(g h)^{1 / 2}}=\frac{2 E\left[\left(\partial u_{L} / \partial y\right)^{2}\right]}{g h} \int_{0}^{x} \rho\left[r,(g h)^{-1 / 2} r\right] d r,
$$

where the shear autocorrelation is

$$
\rho(r, \tau)=\frac{E\left\{\left[\partial u_{L}(x+r, t+\tau) / \partial y\right]\left[\partial u_{L}(x, t) / \partial y\right]\right\}}{E\left[\left(\partial u_{L} / \partial y\right)^{2}\right]} .
$$

For small $x, \rho \approx 1$, so the angle-shear covariance increases linearly with $x$. For large $x$, the angle-shear covariance Eq. (17) attains a constant value, so

$$
-\frac{2 E\left[\theta^{\prime} \partial u_{L} / \partial y\right]}{(g h)^{1 / 2}}=\frac{2 E\left[\left(\partial u_{L} / \partial y\right)^{2}\right] L}{g h},
$$

where

$$
L=\int_{0}^{\infty} \rho\left[r,(g h)^{-1 / 2} r\right] d r
$$

is a decorrelation distance for the shear encountered by propagating swell [although Eq. (14) remains valid only while wave angles remain small].

Substituting Eq. (17) into Eq. (15) and integrating gives (after some manipulation)

$$
E\left[\theta^{\prime 2}(x)\right]=\frac{2 E\left[\left(\partial u_{L} / \partial y\right)^{2}\right]}{g h} \int_{0}^{x}(x-r) \rho\left[r,(g h)^{-1 / 2} r\right] d r .
$$

For small $x$

$$
E\left[\theta^{\prime 2}(x)\right]=\frac{E\left[\left(\partial u_{L} / \partial y\right)^{2}\right] x^{2}}{g h},
$$

so the standard deviation (variance) of the wave angle increases linearly (quadratically) with $x$. In this limit, an initially unidirectional wave would, after propagating $50 \mathrm{~m}$ in 2-m water depth with low-frequency velocity fluctuations of magnitude $0.20 \mathrm{~m} \mathrm{~s}^{-1}$ and alongshore wavelength $100 \mathrm{~m}$, develop a wave angle standard deviation of roughly $5^{\circ}$, comparable to the observations (see section 5 for more detail). For large propagation distances $x$,

$$
E\left[\theta^{\prime 2}(x)\right]=\frac{2 E\left[\left(\partial u_{L} / \partial y\right)^{2}\right] L x}{g h},
$$

the standard deviation of the wave angle increases as $x^{1 / 2}$, and the random refraction acts to directionally diffuse wave energy. This diffusive limit is assumed by many theories for propagation of light and sound through random media (Sobczyk 1985).

Refraction by the alongshore surface slope leads to similar behavior, as can be shown by a straightforward modification of the above derivation.

\section{Comparison of predicted and observed broadening}

\section{a. Predicted broadening}

Equation (13) predicts the variance of a slowly varying wave angle. A slowly varying wave angle $\tilde{\theta}$ was calculated from observations by replacing the 3-h averaging in Eq. (3) with 20-s averaging (similar results were obtained using 10- and 40-s averaging). As in section $2, \hat{u}$ and $\hat{v}$ were calculated by bandpassing 3-h time series.

The predicted directional broadening caused by lowfrequency motions was evaluated using measurements from two five-element cross-shore arrays, separated by $12 \mathrm{~m}$ alongshore (circles, filled triangles, and filled squares in Fig. 1a). For any variable $X$, let $X^{(j, k)}$ denote a measurement of $X$ at the $j$ th cross-shore element $(j=$ $1-5$ with 1 the most seaward location and 5 the most shoreward) of the $k$ th cross-shore array $(k=1,2)$. The predicted current-generated rate of shoreward broadening equals $-2(g h)^{-1 / 2} E\left[\begin{array}{lll}\theta^{\prime} & \partial u_{L} / \partial y\end{array}\right]$ [Eq. (13)] and is estimated at cross-shore location $j$ as

$$
\xi_{u}^{(j)}=-\frac{\left\langle\left[\tilde{\theta}^{\prime(j, 1)}+\tilde{\theta}^{\prime(j, 2)}\right]\left[u_{L}^{(j, 2)}-u_{L}^{(j, 1)}\right]\right\rangle}{\left[g h^{(j)}\right]^{1 / 2}\left[y^{(j, 2)}-y^{(j, 1)}\right]},
$$

where $\tilde{\theta}^{\prime}=\tilde{\theta}-\langle\tilde{\theta}\rangle$. The shoreward increase in spread caused by the sea surface slope, $\xi_{\eta}^{(j)}$, is equal to $-E\left[\theta^{\prime}\right.$ $\left.\partial \eta_{L} / \partial y\right] / h$ [Eq. (13)] and was calculated by replacing the term $\left[u_{L}^{(j, 2)}-u_{L}^{(j, 1)}\right]$ in Eq. (24) with $\left[\eta_{L}^{(j, 2)}-\eta_{L}^{(j, 1)}\right] / 2$. Time series of the low-frequency motions $\eta_{L}$ and $u_{L}$ were calculated by bandpassing quadratically detrended 3-h time series between 0.001 and $0.050 \mathrm{~Hz}$ and sampling once every $20 \mathrm{~s}$ (i.e., the sampling rate of $\tilde{\theta}$ ). The predicted anomalous broadening as waves propagate between locations $j-1$ and $j$ the integral of $\partial E\left[\theta^{\prime 2}\right] / \partial x$ from $x^{(j-1)}$ to $\left.x^{(j)}\right\}$ is estimated as

$$
\left[\Delta \tilde{\sigma}_{a, \mathrm{pred}}^{(j)}\right]^{2}=\left[\frac{\xi_{u}^{(j)}+\xi_{u}^{(j-1)}}{2}+\frac{\xi_{\eta}^{(j)}+\xi_{\eta}^{(j-1)}}{2}\right]\left[x^{(j)}-x^{(j-1)}\right] .
$$

\section{b. Observed broadening}

The observed standard deviation of $\tilde{\theta}$,

$$
\tilde{\sigma}_{\mathrm{obs}}=\left\langle(\tilde{\theta}-\langle\tilde{\theta}\rangle)^{2}\right\rangle^{1 / 2},
$$


where angle brackets denote a 3-h average, will be compared with the wave angle standard deviation $E\left[\theta^{\prime 2}\right]$ predicted by Eq. (13). The wave angle standard deviation in Eq. (26) is related to the conventionally defined directional spread in Eq. (4) (see section 6). The observed anomalous broadening between cross-shore locations $j-1$ and $j$ is

$$
\Delta \tilde{\sigma}_{a, \mathrm{obs}}^{(j)}=\tilde{\sigma}_{\mathrm{obs}}^{(j)}-\left[\frac{h^{(j)}}{h^{(j-1)}}\right]^{1 / 2} \tilde{\sigma}_{\mathrm{obs}}^{(j-1)} .
$$

\section{c. Comparison}

The observed and predicted anomalous broadening between the two most shoreward locations $(x=160$ and $x=210 \mathrm{~m}$ ) were correlated and had similar magnitudes (Fig. 3a). Farther offshore, the predicted broadening tended to exceed that observed, but both the predicted and observed anomalous broadening were small (root-mean-square predicted and observed anomalous spreads were less than $1^{\circ}$ ).

Refraction by the slowly varying shear of the crossshore current was much larger than refraction by the slowly varying alongshore slope of the sea surface [the cross-shore integral of $\xi_{\eta}^{(j)}$ was scattered around zero, with magnitude always less than $\left(1.4^{\circ}\right)^{2}$, whereas the integral of $\xi_{u}^{(j)}$ usually was positive, with magnitude greater than $\left(1.4^{\circ}\right)^{2}$ for 147 of the 348 3-h time series]. Close to the shore, low-frequency kinetic energy greatly exceeded low-frequency potential energy during storms (Fig. $2 \mathrm{~g}$ ) when much of the anomalous spreading occurred (Fig. 2f), indicating a dominance of shear wave over gravity wave energy (Lippmann et al. 1999). Thus, shear waves appear responsible for most of the observed broadening.

The simplified model for short propagation distances [Eq. (22)], combined with the observed water depth and current shear, overpredicted the anomalous broadening by about $30 \%$ (Fig. 3b), consistent with the overprediction of the angle-shear correlation (set equal to 1 in the simple model). The full and simplified model predictions of the anomalous broadening between the two most shoreward locations were correlated $\left(r^{2}=\right.$ 0.73 , slope $=0.6$, offset $=-0.5^{\circ}$, not shown).

\section{Discussion and conclusions}

Low-frequency surf zone currents refract incoming swell, generating low-frequency wave angle fluctuations. A model for this variable refraction predicted the observed shoreward increase in the variance of slowly varying wave angles.

The variance of slowly varying wave angles was related to the conventional directional spread estimated
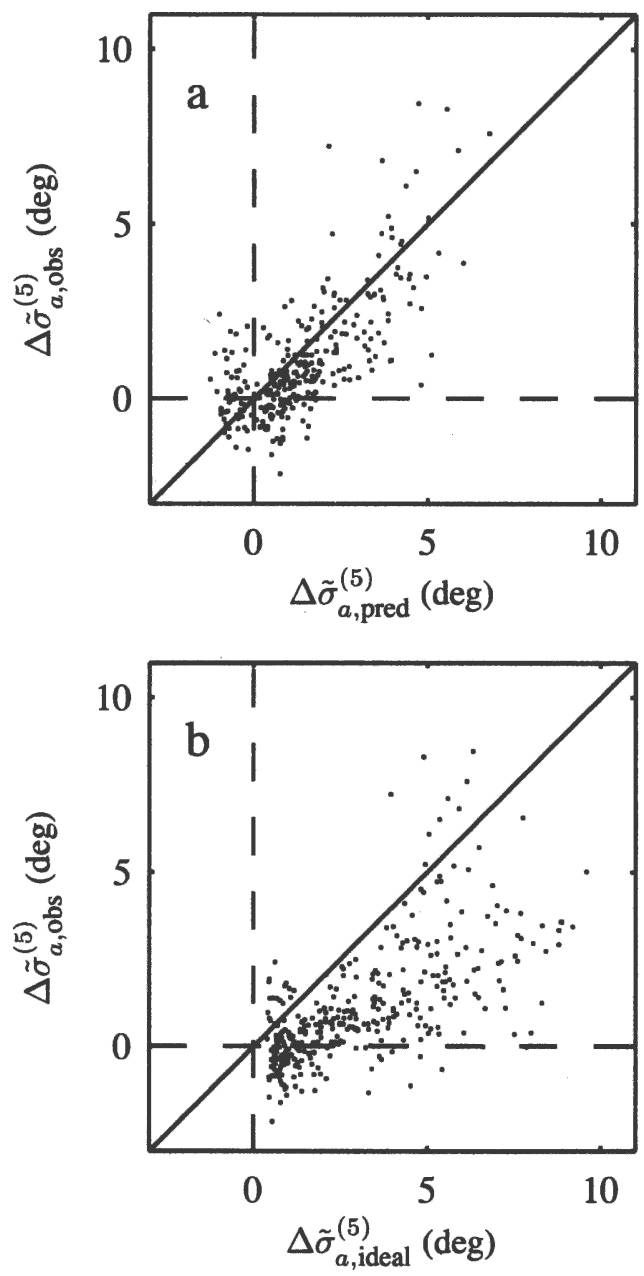

FIG. 3. Anomalous directional broadening between the most shoreward instruments (cross-shore locations 160 and 210 m, Fig. 1). (a) Observed broadening $\left[\Delta \tilde{\sigma}_{a, \text { obs }}^{(5)}\right.$, Eq. (27)] vs full theory prediction $\left[\Delta \tilde{\sigma}_{a, \text { pred }}^{(5)}\right.$, Eq. (25)], $r^{2}=0.5$, slope $=0.8$, and offset $=$ $-0.06^{\circ}$. (b) Observed broadening vs simplified theory prediction $\left[\Delta \tilde{\sigma}_{a, \text { ideal }}^{(5)}\right.$, Eq. (22)], $r^{2}=0.43$, slope $=0.50$, and offset $=-0.47^{\circ}$. The solid line indicates agreement. Each dot represents a 3-h record.

from long time series. Although no theoretical relationship between the two measures of spreading has been derived, the wave angle standard deviations $\tilde{\sigma}$ measured at the shallow and deep instruments (respectively, $\tilde{\sigma}_{\mathrm{s}}$ and $\tilde{\sigma}_{\mathrm{d}}$ ) were correlated with, and about half of, the conventional directional spreads $\bar{\sigma}_{\mathrm{s}}$ and $\bar{\sigma}_{\mathrm{d}}$ (in both cases $r^{2} \approx 0.4$, slope $\approx 0.45$, |offset $<2^{\circ}$ ). The observed anomalous (departure from Snell's law) spreads based on the variability of $\tilde{\theta}$ [Eq. (26)],

$$
\tilde{\sigma}_{\mathrm{a}}=\tilde{\sigma}_{\mathrm{obs}}^{(5)}-\left(\frac{h_{s}}{h_{d}}\right)^{1 / 2} \tilde{\sigma}_{\mathrm{obs}}^{(1)}
$$

and on conventional long records [Eq. (4)], 


$$
\bar{\sigma}_{a}=\bar{\sigma}_{s}-\left(\frac{h_{s}}{h_{d}}\right)^{1 / 2} \bar{\sigma}_{d}
$$

were correlated $\left(r^{2}=0.78\right.$, slope $=0.44$, offset $\left.=1.5^{\circ}\right)$. Given these empirical relationships, the refractively induced increase in $\tilde{\sigma}$ had the right magnitude to explain much of observed anomalous shoreward increase in $\bar{\sigma}$.

When broadening was significant, shear waves dominated over gravity waves at low $(0.001-0.05 \mathrm{~Hz})$ frequencies. Therefore, shear waves likely caused most of the increase in the directional spread of swell crossing the surf zone.

Acknowledgments. Funding was provided by the Office of Naval Research, the National Science Foundation, and the National Ocean Partnership Program. Britt Raubenheimer, Falk Feddersen, the U.S. Army Corps of Engineers Field Research Facility, and the staff of the Center for Coastal Studies made valuable contributions to obtaining the field observations.

\section{APPENDIX}

\section{Wave Angle Evolution}

From Eq. (8),

$$
\left\{\omega-k\left[u_{L} \cos (\theta)+v_{L} \sin (\theta)\right]\right\}^{2}=g k \tanh \left[k\left(h+\eta_{L}\right)\right] .
$$

Substituting the approximations $\sin (\delta)=\delta+O\left(\delta^{3}\right)$, $\cos (\delta)=1+O\left(\delta^{2}\right), \tanh (\delta)=\delta-\delta^{3} / 3+O\left(\delta^{5}\right)$, and the ordering assumptions listed in section 3 into Eq. (A1) leads to

$\left[\omega-k u_{L}+O\left(\varepsilon^{3}\right)\right]^{2}=g k\left[k\left(h+\eta_{L}\right)-\frac{(k h)^{3}}{3}+O\left(\varepsilon^{5}\right)\right]$.

Dividing Eq. (A2) by $k^{2}$ gives

$$
c^{2}\left(1-\frac{2 u_{L}}{c}\right)=g h\left[1+\frac{\eta_{L}}{h}-\frac{(k h)^{2}}{3}\right]+O\left(\varepsilon^{3}\right),
$$

where the phase speed $c=\omega / k$. Multiplying Eq. (A3) by $1+2 u_{L} / c$ gives

$$
c^{2}=g h\left[1+\frac{2 u_{L}}{c}+\frac{\eta_{L}}{h}-\frac{(k h)^{2}}{3}\right]+O\left(\varepsilon^{3}\right) .
$$

Equation Eq. (9) follows from Eq. (A4) and the approximation $(1+\delta)^{1 / 2}=1+\delta / 2+O\left(\delta^{2}\right)$.

Transforming Eq. (7) into cross- and alongshore coordinates yields

$$
\frac{\partial \theta}{\partial x}=\frac{1}{c}\left[\tan (\theta) \frac{\partial c}{\partial x}-\frac{\partial c}{\partial y}\right]-\tan (\theta) \frac{\partial \theta}{\partial y} .
$$

Substituting Eq. (9) and the approximation $\tan (\theta)=\theta$ $+O\left(\theta^{3}\right)$ into Eq. (A5) yields

$$
\begin{aligned}
\frac{\partial \theta}{\partial x}= & \frac{\theta}{h^{1 / 2}} \frac{\partial h^{1 / 2}}{\partial x}-\frac{\partial}{\partial y}\left[\frac{u_{L}}{(g h)^{1 / 2}}+\frac{\eta_{L}}{2 h}-\frac{(k h)^{2}}{6}\right]-\theta \frac{\partial \theta}{\partial y} \\
& +O\left(\varepsilon^{3}, \varepsilon^{2} \partial h / \partial x\right) .
\end{aligned}
$$

Equation (10) follows from Eq. (A6) because the dispersive term (third term in square brackets) is negligible $\left[(k h)^{2}=\omega^{2} h / g+O\left(\varepsilon^{4}\right)\right.$, and $\partial \omega / \partial y$ is negligible $]$.

\section{REFERENCES}

Bowen, A. J., and R. A. Holman, 1989: Shear instabilities of the mean longshore current, 1. Theory. J. Geophys. Res., 94, $18023-18030$.

Dean, R. G., and R. A. Dalrymple, 1991: Water Wave Mechanics for Engineers and Scientists. World Scientific, $353 \mathrm{pp}$.

Elgar, S., T. H. C. Herbers, and R. T. Guza, 1994: Reflection of ocean surface gravity waves from a natural beach. J. Phys. Oceanogr., 24, 1503-1511.

—, R. T. Guza, W. C. O'Reilly, B. Raubenheimer, and T. H. C. Herbers, 2001: Wave energy and direction observed near a pier. J. Waterway Port Coastal Ocean Eng., 127, 2-6.

Feddersen, F., R. T. Guza, S. Elgar, and T. H. C. Herbers, 2000: Velocity moments in alongshore bottom stress parameterizations. J. Geophys. Res., 105, 8673-8686.

Henderson, S. M., and A. J. Bowen, 2003: Simulations of dissipative, shore-oblique infragravity waves. J. Phys. Oceanogr., 33, 1722-1732.

Herbers, T. H. C., S. Elgar, and R. T. Guza, 1995: Generation and propagation of infragravity waves. J. Geophys. Res., 100, $24863-24872$.

,$- \ldots$, and -1999 : Directional spreading of waves in the nearshore. J. Geophys. Res., 104, 7683-7693.

_, N. R. Russnogle, and S. Elgar, 2000: Spectral energy balance of breaking waves within the surf zone. J. Phys. Oceanogr., 30, 2723-2737.

— M. Orzech, S. Elgar, and R. T. Guza, 2003: Shoaling transformation of wave frequency-directional spectra. J. Geophys. Res., 108, 3013, doi:10.1029/2001JC001304.

Lippmann, T. C., T. H. C. Herbers, and E. B. Thornton, 1999: Gravity and shear wave contributions to nearshore infragravity wave motions. J. Phys. Oceanogr., 29, 231-239.

Longuet-Higgins, M. S., 1957: The statistical analysis of a random, moving surface. Philos. Trans. Roy. Soc. London, 249A, 321387.

Oltman-Shay, J., P. A. Howd, and W. A. Birkemeier, 1989: Shear instabilities in the mean longshore current, 2, field observations. J. Geophys. Res., 94, 18 031-18 042.

Özkan-Haller, H. T., and Y. Li, 2003: Effects of wave-current interaction on shear instabilities of longshore currents. $J$. Geophys. Res., 108, 3139, doi:10.1029/2001JC001287.

Sobczyk, K., 1985: Stochastic Wave Propagation. Elsevier, 248 pp.

Taylor, G. I., 1921: Diffusion by continuous movements. Proc. London Math. Soc., 20, 196-212. 